\title{
INVESTIGATION OF REDOX PROCESSES AT SEMICONDUCTOR ELECTRODE LIQUID JUNCTIONS
}

Report on Research Activities

July 15, 1987 - February 14, 1991

Carl A. Koval, Principal Investigator

The Regents of the University of Colorado

Boulder, CO 80309

Prepared August, 1990 for

The U.S. Department of Energy

Agreement No. DE-FG02-84ER13247

\section{DISCLAIMER}

\begin{abstract}
This report was prepared as an account of work sponsored by an agency of the United States Government. Neither the United States Government nor any agency thereof, nor any of their employees, makes any warranty, express or implied, or assumes any legal liability or responsibility for the accuracy, completeness, or usefulness of any information, apparatus, product, or prucess disclosed, or represents that its use would not infringe privately owned rights. Reference herein to any specific commercial product, process, or service by :rade name, trademark, manufacturer, or otherwise does not necessarily constitute or imply its endorsement, recommendation, or favoring by the United States Government or any agency thereof. The views and opinions of authors expressed herein do not necessarily state or reflect those of the United States Government or any agency thereof.
\end{abstract}

\section{MASTER}

DISTRIBUTION OF THIS DOCUMENT IS UNLIN 


\section{TABLE OF CONTENTS}

Section

Page(s)

I. Research Areas, Goals and Accomplishments 1

II. Reactions of Hot Electrons at p-InP / Acetonitrile Interfaces $2-5$

A. Reduction of Copper (II,I) Complex lons

B. Reduction of Alkyl Tin Halides

III. Theory of Electron Transfer at the Semiconductor/Electrolyte Interface

A. Calculation of Image Potentials

B. Electrical Interactions at Interfaces

C. Implications for Reorganization Energies

IV. Measurement of Kinetics at Metal Chalgogenide Electrodes

A. Ideal Polarizable Semiconductor-Solution Interfaces

B. Simultaneous Acquisition of Interfacial Energetics and Kinetic Currents

C. Development of Electrochemical Mini-Cell

V. List of Publications and Manuscripts Acknowledging Support

From DOE Agreement No. DE-FG02-84ER13247

VI. Other Literature Cited 


\section{Research Areas, Goals and Accomplishments}

Our research over the past three years in fundamental aspects of photoelectrochemical celis has been in the following areas: chemical probes for hot carrier processes, electrostatic theory for describing electrical interactions at interfaces, and kinetics of electron transfer at ideal semiconductor solution interfaces $(1-12)$. Dur goal is to achieve a better understanding of dark and photo-induced current flow at the semiconductor electrode / redox electrolyte interface (SEI) so that devices and processes utilizing this interface for solar energy conversion can be developed or improved.

Although significant progress has been made in each of the three areas listed above, our most important accomplishment has been the development of a redox system capable of detecting hot electrons at the $p$ - InP/acetonitrile interface $(7,11)$. The production of copper metal at high doped electrodes is the first example of a product produced by a supra-bandedge reaction in a photoelectrochemical cell. As part of this work, several relevant properties of the $p$ - $\ln P /$ acetonitrile interface were studied which allowed us to develop conditions under which hat carrier reactions can be studied $(4,10)$. We have investigated the mechanism of this reaction and are in the process of extending the research to other systems. Progress in this research area is described in Section II.

Attempts to understand the kinetics of dark electron transfer at the SEI led us to re-examine the assumptions underlying the widely used theoretical model proposed by Gerischer (13-15). In particular, we examined electrostatic theory for the image potential of an ion as a function of distance from the $\operatorname{SEI}(9,12)$. Development of a nonlocal formalism for caizulation of this property led us to believe the prior calculatici of such related properties such as the solvent reorganization energy for electron transier are in crror. This conclusion has been recently been confirmed by Marcus (16). Progress in this research area is described in Section III.

Our group was one of the first to realize that the 2-dimensional metal rhalcogenides (MC) are excellent materials for fundamental studies of electron transfer at the SEI $\left(2,5, C^{\prime}\right)$. One of the chief potential advantages for use of MC's is the formation of semiconductor/liquid junctions with nearly ideal electrochemical properties. A key experimental problem that has hindered research in this area is a lack of ability to create interfaces in electrochemical cells that are free of edgo sites and surface defects and which can be readily renewed by crystal cleavage. To a large extent, we have overcome this problem through the development of a microcell arrangement. Progress in this research area is described in Section IV. 


\section{Reactions of Hot Electrons at $p-\operatorname{InP} /$ Acetonitrile Interfaces}

\section{A. Reduction of Copper $(11,1)$ Complex lons}

When a p-type electrode in depletion is illuminated with photons having energies greater than the band gap of the semiconductor, it is generally thought that the excited electrons are thermalized as they travel through the depletion region, ultimately being ejected into the solution at the energy of either the conduction band edge or of surface states within the bandgap (13). Thermalization represents one of the greatest contributions to power loss in photoelectrochemical cells (PEC's). In hot carrier processes, the electron is ejected with a conservation of a greater fraction of the initial photon energy. Prior to our work, hot carrier effects were predicted to occur in photoelectrochemical systems and there was experimental evidence by Nozik and co-workers that hot carrier effects resulted in enhanced photocurrents in III-V semiconductor system (17). However, no distinct chemical product produced by hot carriers had been reported.

We have used irreversible chemical reactions to trap hot electrons produced at the $p-\operatorname{InP}$ acetonitrile interface $(7,11)$. One example of such a probe is $\mathrm{Cu}(1)$ (transdiene $)^{+}$. The $\mathrm{Cu}(1)$ complex can be reduced by one electron at potentials significantly negative of the InP conduction band edge. This irreversible reaction results in plating of copper metal on the surface of the electrode. Anodically stripping this film is a highly sensitive means of determining the exact amount of $\mathrm{Cu}(0)$ product produced via reaction with hot electrons. Supra-band edge production of $\mathrm{Cu}(0)$ occurs at high doped $p-\ln P$, but was not observed at low doped samples. For the high doped electrodes, hot carrier quantum yields of $0.30 \%$ were measured initially, but these measurements represent a lower limit, sirice the hot carrier probe reaction must compete with other processes (7).

The ability to detect hot electron reactions by our procedure is critically dependent on the ability to attain stable, known interfacial energetics under illumination with up to $0.8 \mathrm{~V}$ of band bending. For this reason we undertook a comprehensive investigation of etched $p-\operatorname{In} P$ interfaces (10). The physical and chemical composition of $p$-InP surfaces prepared with $\mathrm{HCl}$ and $\mathrm{Br}_{2} / \mathrm{NH}_{3}(\mathrm{aq})$ etches were investigated using SEM and XPS, and the interfacial energetics were studied using capacitance and open circuit photovoltage measurements. The $\mathrm{Br}_{2} / \mathrm{NH}_{3}(\mathrm{aq})$ etch produces a microscopically smooth surface with a surface layer containing less than a monolayer of impurities, composed primarily of $\operatorname{lnPO} \mathrm{P}_{4}$ with some $\ln (\mathrm{OH})_{3}$ and some adsorbed hydroxyl species. The $\mathrm{HCl}$ etch prociuces a rough, crystalline surface 
with a 1-2 monolayer indium rich surface layer which is heavily hydrated and contains $\mathrm{In}_{2} \mathrm{O}_{3}$ or $\ln (\mathrm{OH})_{3}$ and adsorbed hydroxyl species.

Capacitance measurements of the $\mathrm{p}$-InP/acetonitrile interface reveal that the interfacial energetics of $\mathrm{Br}_{2} / \mathrm{NH}_{3}(\mathrm{aq})$ etched electrodes are controlled by filling of empty surface states by solution redox couples. The $\mathrm{Br}_{2} / \mathrm{NH}_{3}(\mathrm{aq})$ etch yields a $\mathrm{p}-\ln \mathrm{P}$ surface which allows efficient electron transfer to the solution resulting in a deep depletion energetic condition under extreme reverse bias conditions. These studies reveal that the behavior of $\mathrm{p}$-InP photoelectrochemical cells is determined largely by the method of surface pretreatment. Even when the surface films are only a few monolayers thick, their effect on the surface energetics are dramatic.

Additional experiments directed towards understanding the mechanistic aspects of the supra-band edge reduction of copper(I)(trans-diene) ${ }^{+}$to copper metal were also undertaken (11). Results for experiments performed over a much wider range of conditions ellucidated a number of features of the process. Lower limits for hot electron quantum efficiencies up to $2.6 \%$ were measured at high doped $p \cdot \ln P$ photoelectrodes. A strong dependence of the quantum efficiency on the acceptor concentration confirmed that electron transfer to $\mathrm{Cu}(\mathrm{I})$ (trans-diene)+ is the rate determining step in the process. The reduction of $\mathrm{Cu}(\mathrm{I})($ trans-diene) + to $\mathrm{Cu}(0)$ is not mediated by the supporting electrolyte, and copper metal does not significantly catalyze the reduction of the copper complex. No products of hot electron reductions are observed at low doped $p$-InP electrodes under any conditions. Electrolysis experiments at $n$-inP electrodes indicate that only $7 \%$ of the $\mathrm{Cu}(\mathrm{I})\left(\right.$ trans-diene) ${ }^{+}$ which is reduced results in surface-bound copper metal. This result indicates that the true hot carrier quantum efficiency may be as high as $2.6 / 0.07=37 \%$. Based on this possibility, we are attempting to investigate other probe systems that produce soluble reduction products.

\section{B. Reduction of Organotin Halides}

The reduction of the organotin halides was developed to overcome the limitations of the $\mathrm{Cu}(\mathrm{l})($ trans-diene)+ hot carrier probe. One problem with the $\mathrm{Cu}$ complex lies in it's inability to trap hot electrons efficiently in a form that can be easily detected. This results in measurements of the hot carrier quantum efficiency that may be substantially lower than the true values. Another problem of the $\mathrm{Cu}(\mathrm{l})$ complex acting as a hot carrier probe includes plating of copper metal on the surface of the semiconductor, which at surface coverages greater than $5 \%$, can lead to significant effects on the interfacial energetics of the semiconductor electrode / redox electrolyte 
interface. The final problem with the Cu(I) hot carrier probe system is it's incapacity to independently vary the concentrations of the hot electron acceptor and the thermalized electron acceptor or their relative reduction potentials.

The reduction of organotin halides has been investigated utilizing rotating ring disk electrode (RRDE) voltammetry. This technique should allow us to observe the product of the hot carrier reaction occurring at the disk to be detected at the ring via the following mechanism (18):

Disk: $\mathrm{R}_{3} \mathrm{SnCl}+$ hot $\mathrm{e}^{-} \rightleftharpoons \mathrm{R}_{3} \mathrm{SnCl} \cdot \bullet$ $\mathrm{R}_{3} \mathrm{SnCl} \cdot \bullet \rightarrow \mathrm{R} 3 \mathrm{Sn} \cdot+\mathrm{Cl}^{-}$(fast)

$2 \mathrm{R}_{3} \mathrm{Sn} \cdot \rightarrow \mathrm{R}_{3} \mathrm{SnSnR} \mathrm{R}_{3}$ (fast)

Ring: $\mathrm{R}_{3} \mathrm{SnSnR}_{3} \rightarrow 2 \mathrm{R}_{3} \mathrm{Sn}_{+}+2 \boldsymbol{\theta}^{-}$

Preliminary research has involved construction and shaping of the indium phosphide disk assembly. $\mathrm{N}$-type InP was utilized first because at negative electrode Dotentials the electrode enters the accumulation condition and behaves like a metal. This allows us to investigate the electrochemistry of a number of related organotin halide compounds using cyclic voltammetry, establishing reduction potentials and reversibility. Secondly, we were able to estimate the minimum amount of organotin halide produced at the disk which will still allow detection of the organotin dimer at the ring. The result of this experiment showed that we must produce at least $8.21 \mu \mathrm{A} / \mathrm{cm}^{2}$ at the disk in order to observe $2.60 \mu \mathrm{A} / \mathrm{cm}^{2}(\mathrm{~S} / \mathrm{N}=3)$ at the ring at $1000 \mathrm{rpm}$.

An additional experiment that better represents a hot photoreduction was implemented by poising a solution of cobalticene $e^{+, 0}([\mathrm{COB}+] /[\mathrm{COB}]=90 / 10)$. The potential of the $n$-InP disk electrode is held at the rest potential of the solution and the potential of the $\mathrm{Pt}$ ring is held at a potential positive enough to oxidize the reversible couple. Hence the $n$-inP disk is placed at zero current and the ring is oxidizing $\mathrm{COB}$ at a mass transport limited rate. The disk was then slowly $(0.5 \mathrm{mv} / \mathrm{sec})$ scanned negative and the subsequent increase in cathodic current at the disk was observed as an increase in anodic current at the ring. This was done in order to simulate the light and dar'x conditions of a hot carrier experiment in which we must be able to detect the current due to the oxidation of the organotin dimer on top of the limiting anodic current due to the oxidation of the thermal electron acceptor. The 
results of this experiment showed that when we produced $48.0 \mu \mathrm{A} / \mathrm{cm}^{2}$ at the disk we were able to observe $16.4 \mu \mathrm{A} / \mathrm{cm}^{2}$ of additional current density at the ring.

One final experiment was directed towards illustrating the performance of the InP RRDE relative to a standard RRDE. This was completed by comparing the collection efficiency of the $n$-InP disk RRDE with the collection efficiency of a similar sized platinum disk RRDE. Results of this experiment showed a $22.6 \%$ and a $21.7 \%$ collection efficiency for the $n-\operatorname{In} P$ and platinum RRDE, respectively. The theoretical collection efficiency for a RRDE with the same dimensions as the InP and platinum RRDE was calculated to be $22.5 \%$.

Based on these results, we should be able to detect and quantify hot electrons injected into the solution utilizing the $p-\operatorname{InP} / \mathrm{Pt}$ RRDE if the ratio of hot electrons to total photocurrent exceeds $1 \%$.

\section{Theory of Electron Transier at the Semiconductor/Electrolyte Interface}

\section{A. Calculation of Image Potentials}

The semiconductor electrode-electrolyte interface (SEI) has unique properties that result in kinetics for electron transfer processes which differ from those obiserved at the metal electrode-electrolyte interface (MEI) or in homogeneous systeris (19). Unfortunately, the gap between experiment and theoretical understanding for the SEI is presently large. Existing theories often predict currents that disagree with experiment by many orders of magnitude, even in cases where it is difficult to believe large experimental error exists. Many well-established results from solid-state and surface physics are almost universally neglected in theories describing fundamental properties at solid/electrolyte interfaces. In an initial attempt to obtain some insight into this problem, we focused on a SEI energetic parameter that has a much more complex interpretation than is generally attributed to it: the image potential energy for an ion.

Our initial paper employs a non-local electrostatic approach to calculate the image potential at the SEI. The Korynyshev group has published numerous papers dealing with non-local effects at interfaces (20-22); the same specular reflection model was employed by us. Both the semiconductor and the electrolyte posses structure that is important at short distances so that wavevector dependence of the dielectric response was expected. The wavevector, $q$ equals $2 \pi / \lambda$ where $\lambda$ is the 
wavelength of the disturbance. This response has been thoroughly investigated for semiconductors and was modeled phenomenologically for the solvent. Both the semiconductor and the electrolyte exhibited a split personality in that features of both dielectrics and conductors were present for each medium. In some instances one aspect of the media's nature dominated, but the usual case was intermediate. Nonlocal electrostatics allowed the two characteristics to be incorporated in a single formulation in which they communicated with each other by their common wavevector.

The image potential was calculated by means of contour integration using the calculus of residues. Many values of physical constants for the SC and solvent media were used. Water, acetone, and dimethylformamide solvents were simulated using the appropriate constants. A 1-1 electrolyte at $0.0001,0.01$, and $0.1 \mathrm{M}$ was simulated using a Debye formalism. The results reveal a virtual continuum of possible behavior for the image potential as a function of distance from the electrode.

\section{B. Electrical Interactions at Interfaces}

Classical fluid statistical mechanical theories, and analogous theories traditionally associated with solid state physics, were used to examine electrostatics in the weak coupling limit for an interface of Coulombic systems (12). The imaging potentials of the solid state approaches were shown to be equal in form to those of liquid state approaches for simple models which rely on the specular reflection ansatz. This equivalence also held for the full (screened) electrostatic potentials. The simple model equations were shown to be related to general equations of both the liquid and solid state formalisms. Relationships were identified that, on a simple level, serve to unify very general theories of many body interfacial electrical interactions. The relationships are especially pertinent to the electrochemical interface.

\section{Implications for Reorganization Energies}

A zero order approximation to the SEI point charge image potential assumes that the semiconductor and electrolyte can be treated as structureless classical dielectrics with static dielectric constants $\varepsilon_{\text {semi }}^{\circ}$ and $\varepsilon_{\text {solv }}^{\circ}$. For this system the image potential or "interaction energy" is 


$$
W(R)=\frac{\left(e^{2}\right)}{4 \varepsilon_{\text {solv }}^{\circ} R} \frac{\left(\varepsilon_{\text {solv }}^{\circ}-\varepsilon_{\text {semi }}^{\circ}\right)}{\left(\varepsilon_{\text {solv }}^{\circ}+\varepsilon_{\text {semi }}^{\circ}\right)}
$$

where $e$ is the fundamental unit of charge and $R$ is the distance of the point charge from the electrode surface. If $\varepsilon_{\text {semi }}^{0}<\varepsilon_{\text {solv }}^{0}$, the point charge is repelled from the surface and v.v. Thus to zero order, a point charge would typically be repelled from a SEI since $\varepsilon_{\text {semi }}^{o}$ is almost always less than $\varepsilon_{\text {solv. }}^{0}$. In the case of a metal electrode, $\varepsilon_{\text {semi }}^{o}=\varepsilon_{\text {met }}^{0}=\infty$ so the expression converges to $\left(-e^{2} / \varepsilon_{\text {solv }} 4 R\right)$, a simple attractive coulombic potential. This is the result used in the Marcus expression for the distance dependence of reorganization energy (2):

$E_{r}=\left(e^{2} / 2\right)\left(1 / \varepsilon_{\text {op }}-1 / \varepsilon_{\text {solv }}^{o}\right)(1 / a-1 / 2 R)$.

The Kornyshev group's calculations for the solvent reorganization energy $\left(E_{r}\right)$ for the ME:I (22) showed monotonic increases and decreases as the electrode is approached, as well as hybrid behavior. Since the Marcus equations only predicts decreasing $E_{r}$ as the electrode is approached, these results were significant. We have done explicit distance dependent $E_{r}$ calculations for the SEI both assuming a local solvent and assuming full spatial dispersion. Extremes of the parameters were used in the full spatial case to simplify the very difficult mathematics of the SEI. The formulations make direct use of the IP equations. The computations were done for negligible ionic strength since this is the limit the Marcus equation assumes and because of mathematical hardship. Reorganization energy calculations will be presented in full when we submit a more complete formulation for the SEI $E_{\mathrm{r}}$ that takes into account ion polarizability and vibrational contributions. The curv, followed the pattern of the IP curves in the local solvent case. But in the full spatial dispersion case, when the IP is purely attractive, the reorganization energy curves can sometimes be repulsive (monotonically increasing as the electrode is approached). Hybrid curves appear too. We stress that our results for the full spatial dispersion case are preliminary, yet the above results are not expected to change significantly with more sophistication. In particular, there is no reason to believe that 
the qualitative result of increasing reorganization energy near the interface, especially for high ionic strength, should disappear with a more advanced formulation. This repulsive behavior is again in direct opposition to the typically employed Marcus result and may lead to optimum distances of electron transfer greater than the ionic radii, as can be inferred. Recently Marcus has revised his original theory to address the SEI specifically (16). He also concludes the $E_{r}$ for the SEI could be substantially (factor of $2 x$ ) larger than for the MEI.

\section{Measurement of Kinetics at Metal Chalgoganide Electrodes}

\section{A. Ideal Polarizable Semiconductor-Solution Interfaces}

Prior to the 1970's, electrochemical kinetic studies were largely directed towards faradaic reactions occurring at metal electrodes. While certain questions remain unanswered, a combination of theoretical and experimental studies has produced a relatively mature picture of electron transfer at the metal-solution interface (23). Recent interest in photoelectrochemical processes has extended the interest in electrochemical kinetics to semiconductor electrodes (24-26). Despite the pioneering work of Gerischer (13-15) and Mem.ning (26), many aspects of electron transfer kinetics at the semiconductor-solution interface remain coritroversial or unexplained.

Together with B.A. Parkinson, we proposed that a major difficulty in interpreting kinetic current flow at the semiconductor-solution interface lies in the inability of experimentalists to prepare interfaces with ideal and measurable properties (5). In support of this hypothesis, the importance of ideal interfacial properties to metal electrode kinetic studies was briefly reviewed and a set of criteria for ideality of semiconductor-solution interfaces were developed. Finally, the use of semiconcuuting metal dichalcogenide electrodes as ideal interfaces for subsequent kinetic studies was explored.

\section{B. Simultaneous Acquisition of Interfacial Energetics and Kinetic Currents}

Semiconductor electrochemistry (non-illuminated semiconductor electrodes) involves the same physical principles as photoelectrochemistry, but since it does not involve photogenerated carriers and their inevitable recombination rates, it is inherently less complicated. Nonetheless, there are difficulties in obtaining 
meaningful fundamental information, particularly on narrow band gap semiconductor electrodes which are of most interest in photoelectrochemistry. Among the important areas of interest is the understanding of the energetic factors which influence rates of electron transfer across the semiconductor/electrolyte interface. The so-called interfacial energetics (IE), are a primary consideration in understanding interfacial kinetics. The IE relate the energies of the valence and conduction bands with the energy levels associated with solution redox couples. The IE must also contain a spatial description of the electric fields' that exist in the semiconductor (space charge region, SCR) and solution (Helmholtz layer).

Our approach to this problem has been the simultaneous determination of IE during charge transfer measurements (6). This procedure allows the instantaneous state of the electrode surface to be monitored, while charge transfer is occurring. Thus, if band edge movement does occur, it can be detined and used in the interpretation of the current-potential characteristics. We have described the acquisition of interfacial energetics using impedance analysis during the measurement of electron transfer kinetics to metallocene redox couples. Unfortunately, the use of impedance analysis to determine interfacial energetics is not without its own complications. Although impedance analysis is probably the most commonly used method for determination of interfacial energetics, the problems can be so deleterious as to create true skeptics of the validity of the analysis. Our data show that by careful attention to detail and selection of appropriate systems, very accurate and meaningful results can be obtained.

Our measurements were performed on nine single crystal $\mathrm{WSe}_{2}$ electrodes, five n-type and four p-type with doping densities spanning three orders of magnitude (2). $\mathrm{WSe}_{2}$ electrodes have desirable properties which permit accurate impedance analysis. These include exceptional stability to corrosion and a layered lattice structure. Excellent planar electrode surfaces prepared by cleavage of surface layers are maintained even during prolonged experimentation.

In many respects, $W_{S} e_{2}$ represents an ideal material for fundamental investigations of the electrochemistry of narrow band gap semiconductors (5). Our impedance results supported these contentions. These results established the technique of simultaneous interfacial energetic determinations and defended the validity of carefully executed impedance analyses for the determination of interfacial energetics at least in these systems.

Examples of kinetic current-potential curves obtained simultaneously with the interfacial energetics were presented. While these curves were not analyzed in 
detail, it is clear that even in these well defined systems, several electron transfer mechanisms may be operative and that traditional expressions are not adequate for quantitatively predicting the magnitude of the current.

\section{Development of Electrochemical Mini-Cell}

Although metal dichalcogenides (MDC's) exhibit near-ideal behavior at the cleaved surface, edge sites and defects have much different characteristics and must be avoided. Past studies have enclosed the edges of the electrodes by sealing the crystals in epoxy (Figure 1). Unfortunately this eliminates the ability to prepare fresh surfaces via cleavage. This method further requires very large, defect-free crystals which are non-trivial to synthesize. Once one of these electrodes becomes damaged or degrades with use, it must be discarded. Other possible problems include "leakage currents" escaping through the epoxy and uneven doping through the depth of the crystals.

Because on these concerns, we have developed a novel mini-electrochemical cell to circumvent permament sealing of the crystal edges (Figure 1). This design allows experiments to be performed in a single drop of electrolyte on the surface of the working electrode. By placing the drop on a defect-free zone of a MDC electrode, the rear-ideal interface is established and the solution is isolated from the edge sites. A given electrode can now be recleaved many times providing fresh surfaces between experiments. The doping profile may be investigated and leakage currents through the epoxy are no longer a factor.

The minicell body consists of a teflon $T$-joint for $1 / 16$ " tubing with a solution inlet at the sidearm. The solution passes through a small glass support tube that holds the drop against the working electrode. A $1 \mathrm{~mm}$ diameter Pt tube enters the Tjoint at the bottom to provide counter electrode. A platinum wire sealed in GC capillary tubing runs through the counter electrode and terminates near the working electrode to act as a quasi-reference electrode. The reference potential is conveniently established by using poised redox solutions. The counter electrode is equidistant from the working surface and yet is removed enough to isolate counter electrode products. This design required no special parts and was easily constructed.

However, some special considerations had to be addressed. A solventwetted canopy was added to to prevent evaporation at the drop of electrolyte. Since each drop has a unique size, an internal means for area measurement was developed. By adding a second, reversible redox couple (oxidized species only) with 


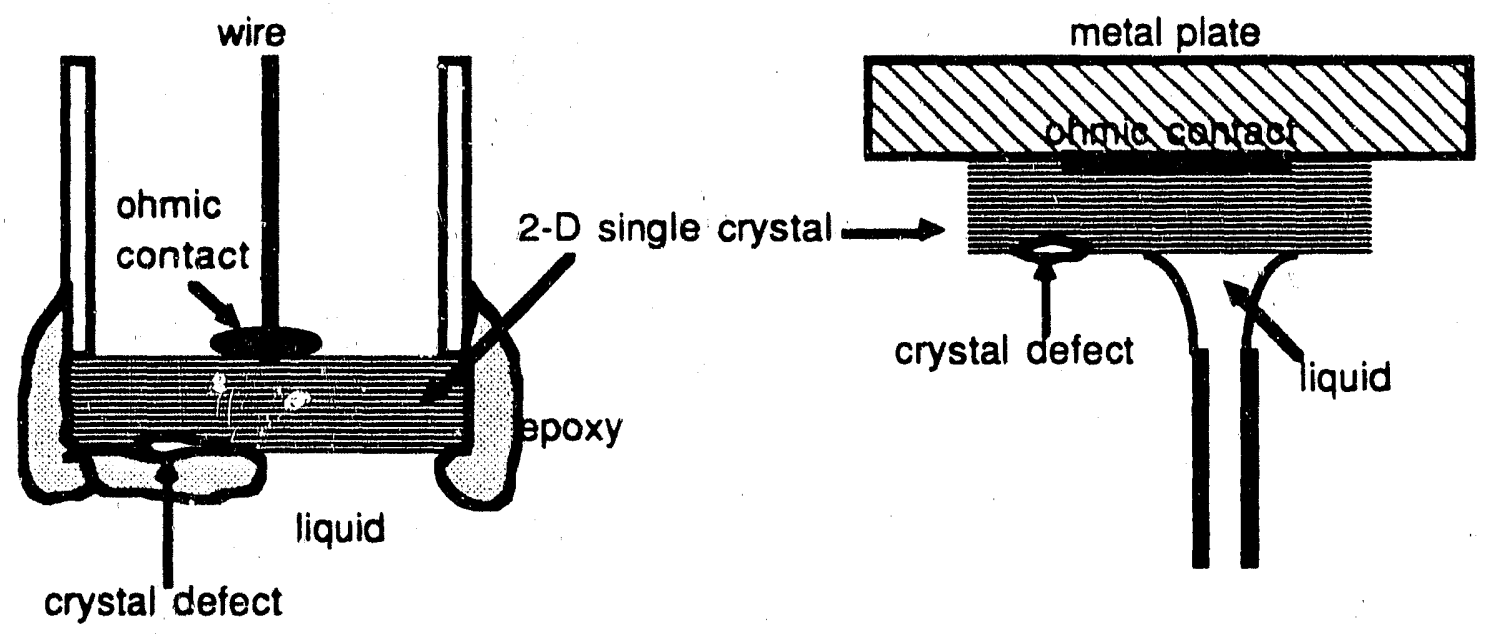

Figure 1. Normal procedure for sealing/masking electrodes (left) and use of mini electrochemical cell (right).

a much more negative reduction potential, the area can be ascertained from the cyclic votammogram of this species. Better accuracy and reproducibility of the area measurement has been obtained by converting the CV to its semiintegral. The use of cobaltocenium hexafluorophosphate extends this procedure to $\mathrm{WSe}_{2}$ since the $\mathrm{CV}$ wave for this cuiple appears while the semiconductor is in accumulation (metallic behavior). The stability of the drop can be checked by following the cell response to a small amplitude ( $<10 \mathrm{mV}$ ) AC voltage. A stable $A C$ current from the cell indicates that the drop size has stabilized. Monitoring of the $A C$ current with an oscilloscope showed a normal sine wave with no perturbations.

The performance of the minicell was studied mostly through cyclic voltammetry at metal electrodes in acetonitrile solutions with ferroceneolt, solution along with cobaltocenium, and $\mathrm{TBABF}_{4}$ electrolyte. Generally, the potential of the waves were found to be stable within a few millivolts while peak heights varied by about $1 \%$ for repeated scans at a given drop. However, changes in bulk concentrations within the drop could occur if too much charge was passed thus leading to potential drift and other anomalies. Fortunately, most of our experiments involve low currents and short times so this problem can be avoided.

The characterization of the cell performance also pointed out the importance of the reference electrode position. Proper potential control has only been obtained 
with the reference tip even with the support tube exit near the working electrode. CV's taken with the reference tip within the body of the support tube were quite distorted.

The use of semiintegration has also revealed the existence of a small thinlayer effect within the minicell. Small peaks found in the semiintegral voltammograms suggest that the extreme edges of the drop are not subject to semi-infinite linear diffusion (27). Although quantitative treatments are difficult, it appears that this effect is small and involves the complete reaction of a species in the thin edges of the drop. Again, for experiments involving small currents this anomaly is not significant.

The minicell has been used to obtain Mott-Schottky plots at WSe2. Flatband potentials and doping densities obtained with the minicell compare quite well with data obtained in macrocells using crystals from the sarne synthesis batch. The minicell has also been used for aqueous experiments. By using $\mathrm{KCl}$ electrolye and a $\mathrm{Ag}$ reference wire etched in $\mathrm{HCl}$, a normal reference half-cell potential was established. It was then possible to perform cyclic voltammetry on single-species redox couples.

Kinetic data obtained so far with the minicell agrees remarkably well with macrocell data. Previously, a jet electrode apparatus (large mass transfer and currents) was used to obtain Tafel parameters for several $\mathrm{WS}_{2}$ electrodes immersed in different metallocene solutions. These experiments showed metallocenes with EO"s deep in the bandgap gave Tafel plots completely inconsistent with theory. Specifically, the forward and reverse exchange current densities did not match and the reverse slopes ( $\mathrm{mV} / \mathrm{log} \mathrm{j}$ ) were too small. Data obtained with the minicell showed the same trends. Below are some specific comparisons of data obtained at $\mathrm{WSe}_{2}$ crystals (from the same growth batch) obtained in the jet electrode and minicell configurations:

JET ELECTRODE, 1987

$1.0 \mathrm{mM}$ fer/fert

$E_{f b}=-695 \pm 3 \mathrm{mV}$

$N_{D}=2.61 \pm .02 \mathrm{E} 16 \mathrm{~cm}^{-3}$

MINICELL, 1990

$2.0 \mathrm{mM}$ fer/fert

$E_{\text {fb }}=-708 \pm 13 \mathrm{mV}$

$N_{D}=1.9 \pm .4 \mathrm{E}_{16 \mathrm{~cm}^{-3}}$

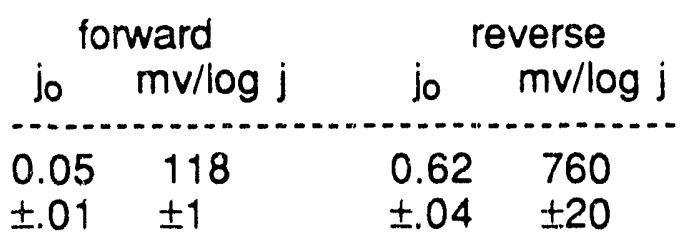

$\begin{array}{llll}0.16 & 117 & 0.51 & 620\end{array}$

$\pm .06 \pm 4 \quad \pm .08 \quad \pm 180$ 
Through these experiments we have optimized the construction and have demonstrated the viability of this minicell to perform electrochemical kinetic measurements. Besides a wide range of experiments at $\mathrm{WSe}_{2}$, this cell may be adaptable to temperature dependance experiments and interfacing with STM. 


\section{List of Publications and Manuscripts Acknowledging Support From DOE Agreement No. DE-FGO2-84ER13247}

(Copies of publications 3 through 11 included in the Appendix)

"1. C.A. Koval, R.M. Gustafson, C. M. Reidema, "The Use of Interrial Standards for the Measurement of Reaction Entropies," Inorg. Chem. (1987) 26, 950-952.

"2. C.A. Koval, J.B. Olson, "Preparation and Electrochemical Characterization of WSe 2 Electrodes Having a Wide Range of Doping Densities," J. Electroanal. Chem. (1987) 234, 133-143.

*3. C. A. Koval, J. B. Olson, "Simple Jet Electrodes for Kinetic and Synthetic Purposes." Anal. Chem. (1988) 60, 88-90.

4. P. R Segar, C. A. Koval, "Interfacial Energetics of the p-InP Acetonitrile Interface" J. Electrochem. Soc. (1988) $135,2655-2656$.

5. C.A. Koval, J.B. Olson, B.A. Parkinson, "Ideal Polarizable Semiconductor-Solution Interfaces." ACS Sym Ser. (1988) 378, 438-450.

6. C. A. Koval, J.B. Olson, "Simultaneou's Determination of Interfacial Energetics and Kinetic Currents at the WSe2/Acetonitrile Interface." J. Phys. Chem. (1988) 92 , 6726-6732.

7. C.A. Koval, P.R. Segar, "Photoelectrochemical Reduction of a Cu(I) Complex To Copper Metal By Hot Electrons at p-InP." J. Am. Chem. Soc. (i989) 111, 2004-10.

8. G.N. Erown, John W. Birks, C.A. Koval, "On-Plate Electrochemical Detection for Thin-Layer Chromatography," Analytical Lett. (1989) 22, 507-518.

9. B. B. Smith and C.A. Koval, "An Investigation of the Image Potential at the Semiconductor/Electrolyte Interface Employing Nonlocal Electrostatics," J. Electroanal. Chem. (1990) 277, 43-72.

10: C.A. Koval, P.R. Segar, B. Koel, S. Gebhard, "A Comprehensive Investigation of $\mathrm{HCl}$ and $\mathrm{Br}_{2} / \mathrm{NH}_{3}(\mathrm{aq})$ Etched p-inP Interfaces," J. Electrochem Soc. (1990) 137 , 544-52.

11. C.A. Koval and P.R. Segar, "Mechanistic Aspects of Reductions by Hot Electrons in p-InP/Acetonitrile Photoelectrochemical Cells," J. Phys. Chem. (1990) 94, 203339.

12. B.B. Smith and C.A. Koval, "Some Interrelationships of Inhomogeneous Liquid and Solid State Theory in the Weak Coupling Limit for Coulombic Systems," J. Chem. Phys., submitted.

* submitted or in press at beginning of grant period, July 15, 1987 


\section{Other Literature Cited}

13. Gerischer, H. in "Advances in Electrochemistry and Electrochemical Engineering", ed. Delahay,P. 1:139-232, Interscience: New York, (1961).

14. Gerischer,H., "Physical Chemistry: An Advanced Treatise", ed. Eyring,H., 9:463542, Academic: New York, (1970).

15. Gerischer,H. in "Topics in Applied Physics", ed. Seraphin,B.O., 31: 115-170, (1979).

16. Marcus, R.A. J. Phys. Chem. 94, 1050 (1990).

17. Cooper,G.; Turner,J.A.; Parkinson,B.A.; Nozik,A.J. J. Appl. Phys., 54(11), 6463 (1983).

18. Dessey,R.E.; Kitching,W.; Chivers,T. J. Amer. Chem. Soc., 88(3), 453 (1966).

19. Kobayashi, K.; Aikawa, Y.; Sukigara, M. J. Electroanal. Chem., 134, 11 (1982).

20. Kornyshev, A.A.; Schmickler, W., Vorotyntsev, M.A., Phys. Rev. (B), 8, 5244 (1982).

21. Kornyshev, A.A.; Rubinstitein, A.I. Pinys. Stat. Sol. (B) 84, 125 (1977).

22. Kornyshev, A.A. Electrochim. Act., 26, 1, (1981).

23. Albery, W.J. Electrode Kinetics, Clarendon Press, Oxíord, 1975.

24. Wilson, R.H.; CRC Crit. Rev. Sol. State Mat. Sci. 1980, 10, 1.

25. S. R. Morrison, Electrochemistry at Semiconductor and Oxidized Metal Electrodes, Plenum Press, N.Y., 1980.

26. Memming, R. Electroanal. Chem. 1979, 11, 1.

27. R. Bowling and R. McCreery, Anal.Chem, 60,605 (1988). 


\section{Appendix - DOE Publications 1988-90}

C. A. Koval, J. B. Olson, "Simple Jet Electrodes for Kinetic and Synthetic Purposes." Anal. Chem. (1988) 60, 88-90.

P R. Segar, C. A. Koval, "Interfacial Energetics of the P-InP Acetonitrile Interface" J. Electrochem. Soc. (1988) 135, 2655-2656.

C.A. Koval, J.B. Olson, B.A. Parkinson, "Ideal Polarizable

Semiconductor-Solution Interfaces." ACS Sym Ser. (1988) 378, 438-450.

C. A. Koval, J.B. Olson, "Simultaneous Determination of Interfacial Energetics and Kinetic Currents at the WSe2/Acetonitrile Interface." J. Phys. Chem. (1988) 92, 6726-6732.

C.A. Koval, P.R. Segar, " Photoelectrochemical Reduction of a $\mathrm{Cu}(\mathrm{l})$ Complex To Copper Metal By Hot Electrons at p-InP." J. Am. Chem. Soc. (1989) 111, 2004-10.

G.N. Brown, John W. Birks, C.A. Koval, "On-Plate Electrochomical Detection for Thin-Layer Chromatography," Anaiytical Lett. (1989) 22, 507-518.

B. B. Smith and C.A. Koval, "An Investigation of the Image Potential at the Semiconductor/Electrolyte Interface Employing Nonlocal Electrostatics," J. Electroanal. Chem. (1990) 277, 43-72.

C.A. Koval, P.R. Segar, B. Koel, S. Gebhard, "A Comprehensive Investigation of $\mathrm{HCl}$ and $\mathrm{Br}_{2} / \mathrm{NH}_{3}(\mathrm{aq})$ Etched $\mathrm{p}$-InP Interfaces," J. Electrochem Soc. (1990) 137, 544-52.

C.A. Koval and P.R. Segar, "Mechanistic Aspects of Reductions by Hot Electrons in p-InP/Acetonitrile Photoelectrochemical Cells," J. Phys. Chem. (1990) 94, 2033-39.

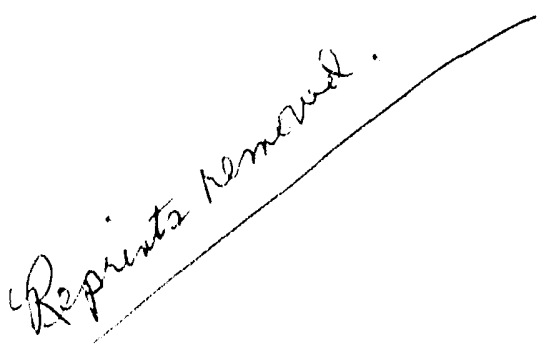



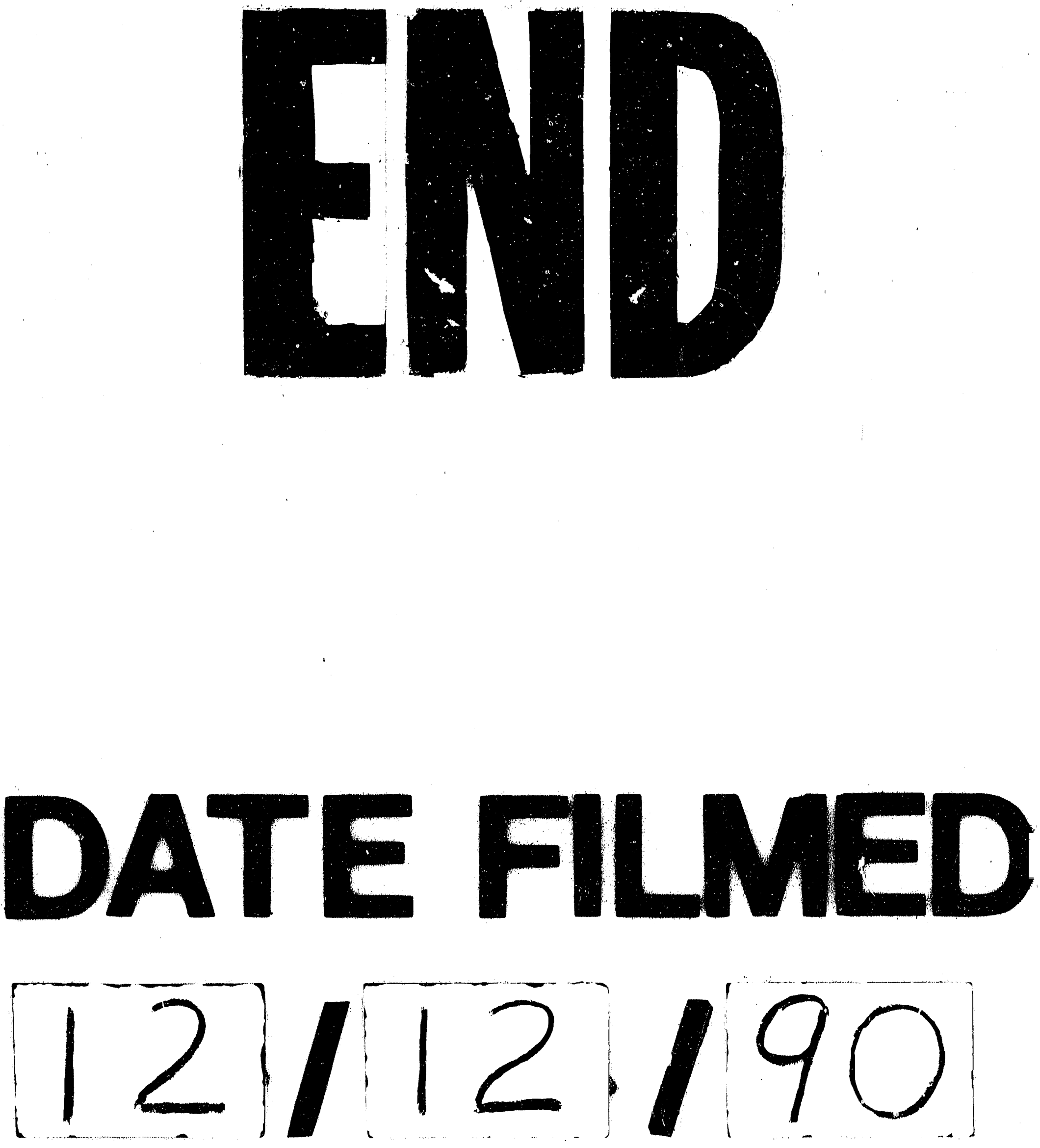


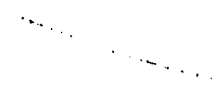

